\title{
A forecasting performance comparison of dynamic factor models based on static and dynamic methods
}

\author{
Fabio Della Marra ${ }^{1 *}$ \\ ${ }^{1}$ Department of Economics, University of Parma, Parma, Italy. \\ *Email address for correspondence: fabio.dellamarra@iispascal.it \\ Communicated by Nicola Bellomo \\ Received on 12 14, 2016. Accepted on 02 16, 2017.
}

\begin{abstract}
We present a comparison of the forecasting performances of three Dynamic Factor Models on a large monthly data panel of macroeconomic and financial time series for the UE economy. The first model relies on static principal-component and was introduced by Stock and Watson $(2002 \mathrm{a}, \mathrm{b})$. The second is based on generalized principal components and it was introduced by Forni, Hallin, Lippi and Reichlin (2000, 2005). The last model has been recently proposed by Forni, Hallin, Lippi and Zaffaroni (2015, 2016). The data panel is split into two parts: the calibration sample, from February 1986 to December 2000 , is used to select the most performing specification for each class of models in a insample environment, and the proper sample, from January 2001 to November 2015, is used to compare the performances of the selected models in an out-of-sample environment. The metholodogical approach is analogous to Forni, Giovannelli, Lippi and Soccorsi (2016), but also the size of the rolling window is empirically estimated in the calibration process to achieve more robustness. We find that, on the proper sample, the last model is the most performing for the Inflation. However, mixed evidencies appear over the proper sample for the Industrial Production.
\end{abstract}

Keywords: Macroeconomic Forecasting, Dynamic Factor Models, Time-domain methods, Frequency-domain methods

AMS subject classification: 62P20

\section{Introduction}

In this paper, a comparative analysis of the forecasting performance of three Large-Dimensional Dynamic Factor Models is presented. As a key feature, Dynamic Factor Models represent each variable in a dataset as the sum of two orthogonal terms: a common component $\chi_{t}$, driven by a reduced (as compared to the number of series in the dataset) number of common factors, and an idiosyncratic component $\xi_{t}$, which represents measurement errors or local features. Among the different versions of the Dynamic Factor Models we selected: 


\section{Fabio Della Marra}

(i) $S W$ model. This time-domain method was introduced in [1], [2]. The factors are estimated by computing static principal components of the variables in the dataset. Let $y_{i t}$ be the variable of the dataset to be forecasted at time $t$, its $h$-step-ahead prediction equation (also called Diffusion Forecast Index) is obtained by regressing $y_{i t+h}$ on the factors and on $y_{i t}$ itself. Lags of the factors and of $y_{i t}$ may be added.

(ii) FHLR model. This frequency-domain method was proposed in [3], [4] and requires the computation of two steps. In a first step, the common component $\chi_{t}$, the idiosyncratic component $\xi_{t}$ and their covariances are estimated using a frequency-domain method introduced in [3] named Dynamic Principal Component. In the second step, the factors are estimated by computing Generalized Principal Components.

(iii) FHLZ model. This frequency-domain method was proposed in [5], [6]. Here, the underlying assumption in (i) and (ii) that the common components span a finite-dimensional space as $n$ tends to infinity is relaxed. The estimation of the parameters is much more complex though.

There exists some literature comparing the forecasting performances of SW and FHLR, but universal consensus still does not seem to have been reached. Theoretically, time-domain methods consider only relations among the variables at the same time, whereas frequency-domain methods exploit leaded and lagged relations among the variables. However timedomain methods require less parameters to be calibrated. Hence they are more robust to misspecification than frequency-domain methods. Empirically, in [7] Boivin and $\mathrm{Ng}$ found that SW generally outperforms FHLR on US macroeconomic data, whereas D'agostino and Giannone in [8] found no relevant differences in the performance of both methods on the whole sample (even though heterogeneity is found in subsamples). In [9] Schumacher found that FHLR outperforms SW on the prediction of the German GDP. The same conclusions are drawn in [10] over the forecasting of Dutch GDP. So far, a systematic comparison of the forecasting performances of SW, FHLR and FHLZ can be found only in [11]. Here, Forni et al. conducted a forecasting exercise on an US macroeconomic dataset, where they took an autoregressive process of order 4 as a benchmark. They showed that FHLZ oupterforms SW, FHLR and the benchmark both for Industrial Production and Inflation during the Great Moderation. In the Great Recession, the forecasting performances of the Industrial Production change dramatically: all factor models are outperformed by the benchmark and SW and 


\section{Forecasting performance comparison of dynamic factor models}

FHLR outperform FHLZ. Hence, Forni et al. concluded that, due to its more dynamical structure, FHLZ tends to be the best performing method in "stationary period", but it loses ground during regime changes. Also, they showed that FHLZ tends to be outperforming on nominal variables and FHLR on real variables.

In this paper, a large macroeconomic dataset consisting of $176 \mathrm{EU}$ macroeconomic and financial time series observed at monthly frequency over the period from February 1986 to November 2015 is used to analyse the forecasting performance of these methods. To achieve stationarity, the series are deseasonalized and transformed. No treatment for outliers is applied.

As in [11], the EU dataset is split into two subsamples. The former, from February 1986 to December 2000, will be used to calibrate the models, i.e. to produce in-sample forecasts of the variables of the EU dataset for several specifications of SW, FHLR and FHLZ. Then, for each class of models, we selected the specification which shows the minimum mean square forecast error (MSFE). These models are then run and compared in the remaining sample, from January 2001 to November 2015.

The paper is structured as follows. In Section 2, the factor models are discussed in detail. In Section 3, the main features of the dataset are illustrated. In Section 4, the calibration process of the models is described. In Section 5, results are discussed and Section 6 concludes.

\section{An overview on Dynamic Factor Models}

Let $\left\{\mathbf{x}_{\mathbf{t}}=\left(x_{1 t} \ldots x_{n t}\right)^{\prime} \mid t=1, \ldots, T\right\}$ be a $n$-dimensional vector of time series, which will be denoted as $\mathbf{x}_{\mathbf{t}}$ for simplicity. $\mathbf{x}_{\mathbf{t}}$ will be assumed to be weakly-stationary, purely non deterministic with zero mean and unit variance. Let us assume that the following decomposition holds:

$$
\mathbf{x}_{\mathbf{t}}=\chi_{t}+\xi_{t} .
$$

The process $\chi_{t}=\left(\chi_{1 t} \ldots \chi_{n t}\right)^{\prime}$ is called common component. It will be assumed that $\chi_{i t}$ is stationary and that $\chi_{i t}$ is costationary with $\chi_{j t}$ for all $i, j=1, \ldots, n$ such that $i \neq j$. The process $\xi_{t}$ is called idiosyncratic component. It will be assumed that $\xi_{i t}$ is stationary and that $\xi_{i t}$ is costationary with $\xi_{j t}$ for all $i, j=1, \ldots, n$ such that $i \neq j$. A distinction between static and dynamic factor models can be made according to the functional form selected for the common component. In static factor models, the common component can be modeled as $\chi_{t}=\boldsymbol{\Lambda} \mathbf{F}_{t}$ where $\boldsymbol{\Lambda} \in \mathbb{R}^{n \times r}$ is called the factor-loading matrix and $\mathbf{F}_{t} \in \mathbb{R}^{r}$ is the vector of the static factors at time 


\section{Fabio Della Marra}

$t$, with $r<<n$. In dynamic factor models, the common component takes into account also the lags of the factors. Hence, the common component can be modeled as $\chi_{t}=\boldsymbol{\Lambda}(L) \mathbf{u}_{t}$. Here $L$ is the lag operator, $\boldsymbol{\Lambda}(L) \in \mathbb{R}^{n \times q}$ is called the factor-loading matrix and $\mathbf{u}_{t} \in \mathbb{R}^{q}$ is the vector of the dynamic factors at time $t$, with $q<<n$. In [12], [13], it is shown that $\mathbf{u}_{t}$ is a orthonormal white noise process. Moreover, fixed a maximum lag order $s$ for the matrix $\boldsymbol{\Lambda}(L)$, a dynamic factor model can be rewritten as a static factor model by staking all of the factors with their lags in a single vector, i.e. by imposing $\mathbf{F}_{t}=\left(\mathbf{u}_{t} \ldots \mathbf{u}_{t-s}\right)$. More details can be found in [14]. This way, it holds that $r<<q<<n$. The three different dynamic factor models for estimating the factors are discussed in the following subsections.

\subsection{The $S W$ model}

In [1], [2], Stock and Watson proposed a static factor model whose components are estimated by means of static principal component. Let $\hat{\boldsymbol{\Gamma}}=T^{-1} \sum_{t=1}^{T} \mathbf{x}_{t} \mathbf{x}_{t}^{\prime}$ be the sample covariance matrix of $\mathbf{x}_{t}$. By computing the eigenvalues of $\hat{\boldsymbol{\Gamma}}$ and stacking them into the matrix $\mathbf{P}=\left(\begin{array}{lll}\mathbf{P}_{1} & \ldots & \mathbf{P}_{r}\end{array}\right)^{\prime}$, with $\mathbf{P}_{i}$ the eigenvector corresponding to the $i$-th largest eigenvalue, we can compute the factors $\hat{\mathbf{F}}_{t}=\mathbf{P}^{\prime} \mathbf{x}_{t}$ The $h$-step ahead SW forecasting equation, also called Diffusion Forecast Index, can be computed by regressing $x_{i t+h}$ on the factors $\hat{\mathbf{F}}_{t}$ and $x_{i t}$. Lags of both $\hat{\mathbf{F}}_{t}$ and $x_{i t}$ may be added. Hence, the Diffusion Forecast Index can be modeled as

$$
x_{i t+h \mid t}=\mathbf{a}_{\mathbf{i}}(L) \hat{\mathbf{F}}_{t}+b_{i}(L) x_{i t},
$$

where $\mathbf{a}_{\mathbf{i}}(L)$ is a $n \times r$ vector of polynomials of degree $\alpha_{i}$ and $b_{i}(L)$ is a scalar polynomial of degree $\beta_{i}$.

\subsection{The FHLR model}

This model was proposed by Forni et al. in [3], [4]. It is articulated in two steps:

(i) Estimation of the common and idiosyncratic component: let $\hat{\boldsymbol{\Gamma}}(k)=$ $T^{-1} \sum_{t=1}^{T} \mathbf{x}_{t} \mathbf{x}_{t-k}^{\prime}$ be the sample autocovariance of $\mathbf{x}_{t}$ at lag $k$. In order to consistently estimate the spectral density matrix of $\mathbf{x}_{t}$, we can compute this estimator $\hat{\boldsymbol{\Sigma}}(\theta)=\sum_{t=-d}^{d} w_{k} \hat{\boldsymbol{\Gamma}}(k) e^{-i k \theta}$ with $w_{k}$ being the weights of a kernel function. By computing the spectral decomposition of $\hat{\boldsymbol{\Sigma}}(\theta)$ for all $\theta$, the spectral density matrix of the common component can be reconstructed by computing $\hat{\boldsymbol{\Sigma}}_{\chi}(\theta)=\hat{\mathbf{P}}(\theta) \boldsymbol{\Lambda}(\theta) \hat{\mathbf{P}}^{*}(\theta)$, where $\boldsymbol{\Lambda}(\theta)$ denotes the diagonal matrix whose entries are the $q$ largest eigenvalues of $\hat{\boldsymbol{\Sigma}}(\theta)$ and 


\section{Forecasting performance comparison of dynamic factor models}

$\hat{\mathbf{P}}(\theta)=\left(\hat{\mathbf{P}}_{1}(\theta) \ldots \hat{\mathbf{P}}_{q}(\theta)\right)$ the matrix whose columns are the corresponding eigenvectors. The spectral density matrix of the idiosyncratic component can be reconstructed by differencing $\hat{\boldsymbol{\Sigma}}_{\xi}(\theta)=$ $\hat{\boldsymbol{\Sigma}}(\theta)-\hat{\boldsymbol{\Sigma}}_{\chi}(\theta)$ Autocovariances at lag $k$ of the common and the idiosyncratic component can be obtained by computing the inverse Fourier transform of their estimated spectral density matrix.

(ii) Estimation of the factors: now, the estimated covariance matrix of the common component and of the idiosyncratic component are used to solve the generalized principal components problem:

$$
\hat{\boldsymbol{\Sigma}}_{\xi}^{0} \mathbf{P}=\hat{\boldsymbol{\Sigma}}_{\chi}^{0} \mathbf{P D}
$$

s.t. $\mathbf{P}^{\prime} \hat{\boldsymbol{\Sigma}}_{\chi}{ }^{0} \mathbf{P}=\mathbf{I}$, where $\mathbf{D}$ is a diagonal matrix whose entries are the $r$ largest eigenvalues of the pair $\left(\hat{\boldsymbol{\Sigma}}_{\xi}^{0}, \hat{\mathbf{\Sigma}}_{\chi}^{0}\right)$ and $\mathbf{P}$ is the matrix containing the corresponding eigenvectors. The first $r$ factors are defined as $\hat{\mathbf{F}}_{t}=\mathbf{P}^{\prime} \mathbf{x}_{t}$

By means of the projections:

$$
\begin{aligned}
\hat{\chi}_{i t+h \mid t} & =\operatorname{Proj}\left[\chi_{i t+h} \mid \hat{\mathbf{F}}_{t}\right] \\
\hat{\xi}_{i t+h \mid t} & =\operatorname{Proj}\left[\xi_{i t+h} \mid \mathbf{x}_{i t} \ldots \mathbf{x}_{i t-p}\right],
\end{aligned}
$$

the $h$-step ahead FHLR forecasting equation can be finally derived as

$$
\chi_{i t+h \mid t}=\hat{\chi}_{i t+h \mid t}+\hat{\xi}_{i t+h \mid t} .
$$

\subsection{The FHLZ model}

This model was proposed by Forni et al. in [5], [6]. Differently from the previous models, here the assumption that the common component spans a finite-dimensional space is relaxed. This model is articulated in the steps listed below:

(i) Estimation of the spectral density matrix $\hat{\boldsymbol{\Sigma}}^{\mathbf{x}}(\theta)$ of $\mathbf{x}_{t}$ : the spectral density matrix $\hat{\boldsymbol{\Sigma}}^{\mathbf{x}}(\theta)$ can be estimated as $\hat{\boldsymbol{\Sigma}}^{\mathbf{x}}(\theta)=$ $\frac{1}{2 \pi} \sum_{k=-R}^{R} \omega_{k} \hat{\boldsymbol{\Gamma}}(k) e^{-i k \theta}$ with $\omega_{k}$ representing the weights of a kernel function.

(ii) Estimation of the spectral density matrix $\hat{\boldsymbol{\Sigma}} \chi(\theta)$ of $\chi_{t}$ : it is obtained by computing the dynamic principal components of $\hat{\boldsymbol{\Sigma}}^{\mathbf{x}}(\theta)$ and then by selecting its $q$ principal components which are associated to the largest eigenvalues. For more details, see [3], [5]. 


\section{Fabio Della Marra}

(iii) Estimation of the autocovariance matrices $\hat{\boldsymbol{\Gamma}}_{k}^{\chi}$ of $\chi_{t}$ : The autocovariances of $\chi_{t}$ are estimated by means of the Wiener-Khinchin-Einstein theorem.

(iv) Estimation of the VAR matrices $\hat{\mathbf{A}}^{k}(L)$ : under general assumptions, the common component admits a unique blockwise autoregressive representation of the form:

$$
\left[\begin{array}{ccccc}
\mathbf{A}^{1}(L) & 0 & \ldots & 0 & \ldots \\
0 & \mathbf{A}^{2}(L) & \ldots & 0 & \ldots \\
\vdots & \vdots & \ddots & & \\
0 & 0 & \ldots & \mathbf{A}^{k}(L) & \ldots \\
\vdots & & & & \ddots
\end{array}\right] \chi_{t}=\left[\begin{array}{c}
\mathbf{R}^{1} \\
\mathbf{R}^{2} \\
\vdots \\
\mathbf{R}^{k} \\
\vdots
\end{array}\right] u_{t}
$$

where $\mathbf{A}^{k}(L) \in \mathbb{R}^{(q+1) \times(q+1)}$ is a polynomial matrix of finite degree and $\mathbf{R}^{k} \in \mathbb{R}^{(q+1) \times q}$. To estimate the VAR matrices $\mathbf{A}^{k}(L)$, the covariances $\hat{\boldsymbol{\Sigma}}^{\chi}(\theta)$ are employed.

(v) Estimation of the matrices $\mathbf{R}^{k}$ and the shock $\mathbf{u}_{t}$ : these estimates can be recovered by applying standard principal components to the process $\mathbf{A}(L) \mathbf{x}_{t}$.

By inverting equation (6) it follows that: $\chi_{t}=\left[\mathbf{A}(L)^{-1}\right] \mathbf{R} \mathbf{u}_{t}=$ $\mathbf{C}(L) \mathbf{u}_{t}=\mathbf{C}_{0} \mathbf{u}_{t}+\mathbf{C}_{1} \mathbf{u}_{t-1}+\ldots$ where $\hat{\chi}_{t} \in \mathbb{R}^{n}$ and $\hat{\mathbf{A}}(L), \hat{\mathbf{R}}, \hat{\mathbf{W}}(L) \in \mathbb{R}^{n \times n}$. The $h$-step ahead FHLZ forecasting equation is reported below:

$$
\chi_{i t+h \mid t}=\mathbf{C}_{h} \mathbf{u}_{t}+\mathbf{C}_{h+1} \mathbf{u}_{t-1}+\ldots .
$$

\section{Description of the dataset}

In this empirical application, a large macroeconomic dataset consisting of $176 \mathrm{EU}$ macroeconomic and financial time series observed at monthly frequency is employed. This dataset contains real variables (import/export price indexes, employment, Industrial Production) and nominal variables (money aggregates, consumer price indexes, wages), asset prices (stock prices and exchange rates) and surveys. Further details can be found in the Appendix. To achieve stationarity, several series are deseasonalized and transformed. No treatment for outliers is applied. In addition to SW, FHLR, FHLZ, the forecasts of an autoregressive process (AR) of order 4 are computed. The dataset is divided in two parts: a calibration sample, ranging from February 1986 to December 2000, which will be employed to select the most performing specification of each model, and a proper sample, ranging 


\section{Forecasting performance comparison of dynamic factor models}

from January 2001 to November 2015, which will be employed to compare the selected specifications of each model. As in [2], [8], to assess the forecasting performances, the variables which are taken into account are the level of the logarithm of the Industrial Production (IP) and the yearly change of the logarithm of the Consumer Price Index (CPI). Forecasts are computed $h$-months ahead, with $h \in\{1,3,6,12,24\}$. For each methods, we employed a rolling-window scheme $[t-l, t]$, whose size $l$ will be determined in the calibration sample. To assess the forecasting performance of each model, the mean-square forecast error (MSFE) is employed as a metric:

$$
\operatorname{MSFE}^{h}(i)=\frac{1}{\left(T_{\text {end }}-h\right)-T_{\text {begin }}+1} \sum_{k=T_{\text {begin }}}^{T_{\text {end }}-h} S F E_{k}^{h}(i),
$$

where $T_{\text {begin }}, T_{\text {end }}$ stand, respectively, for the first and the last date in the dataset and $i \in\{S W, F H L R, F H L Z, A R\} . S F E^{h}$ stands for $h$-step ahead squared forecast error and is defined as $S F E^{h}(i)=\left(y_{t+h \mid T}^{i}-y_{t+h}\right)^{2}$, where $y_{t+h \mid T}^{i}$ is the forecasted value at horizon $h$ of the variable $y_{t}$ by the method $i$ and $y_{t+h}$ is its real value.

\section{Calibration}

The calibration procedure is basically the same as in [11], but is more robust since the size of the rolling window is taken into account as a parameter to be tuned. The calibration sample, ranging from February 1986 to December 2000, will be used to calibrate the methods SW, FHLR, FHLZ. Namely, this portion of the dataset will be used to select the best performing specifications of each class of models. To compare the performances of two different methods, say $\alpha, \beta$, at a certain horizon $h$, the ratio mean-square forecast error (RMSFE) will be computed. Such metric is defined as

$$
\operatorname{RMSFE} E^{h}(\alpha, \beta)=\frac{M S F E^{h}(\alpha)}{M S F E^{h}(\beta)} .
$$

\subsection{Calibration of $S W$}

To produce forecastings by means of equation (2), the following parameters must be calibrated:

(i) the number of static factors $r$ : ranging from 1 to 10. Also, a comparison with Bai \& Ng criterium (BN) with maximum 12 factors has been made. 


\section{Fabio Della Marra}

(ii) the degree $\alpha$ of $\mathbf{a}(L)$ : ranging from 1 to 10 .

(iii) the degree $\beta$ of $b(L)$ : ranging from 0 to 10 .

(iv) the size $l$ of the rolling window: ranging from 5 to 12 years.

By selecting the values of the parameters which guarantee the lowest mean RMSFE, the chosen configuration for the IP is the following:

$$
(r, \alpha, \beta, l)=(4,1,0,7) .
$$

Instead, the chosen configuration for the CPI is the following:

$$
(r, \alpha, \beta, l)=(4,1,9,12) .
$$

\subsection{Calibration of FHLR}

To produce forecastings by means of equation (5), the following parameters must be calibrated:

(i) the number of static factors $r$ : ranging from 1 to 10. Also, a comparison with Bai \& Ng criterium (BN) with maximum 12 factors has been carried out.

(ii) the number of dynamic factors $q$ : ranging from 0 to 10. Also, a comparison with Hallin-Liska criterium (HL) with maximum 12 factors has been carried out.

(iii) the type of kernel $k$ : ranging in the set \{Triangular, Rectangular, Parzen, Gaussian, Exponential, Cosine, Tukey, Hann $\}$.

(iv) The lag window $d$ for spectral density estimation: ranging in the set $\{25,35,40\}$.

(v) the size $l$ of the rolling window: ranging from 5 to 12 years.

By selecting the values of the parameters which guarantee the lowest mean RMSFE, the chosen configuration for the IP is the following:

$$
(r, q, k, d, l)=(4,3, \text { Tukey }, 25,12) .
$$

Instead, the chosen configuration for the CPI is the following:

$$
(r, q, k, d, l)=(1,3, \text { Cosine }, 25,7) .
$$




\section{Forecasting performance comparison of dynamic factor models}

\subsection{Calibration of FHLZ}

To produce forecastings by means of equation (7), the following parameters must be calibrated:

(i) the number of dynamic factors q: ranging from 1 to 5. Also, a comparison with Hallin-Liska criterium has been carried out.

(ii) the type of kernel $k$ : ranging in the set \{Triangular, Rectangular, Parzen, Gaussian, Exponential, Cosine, Tukey, Hann\}.

(iii) the lag window $d$ for spectral density estimation: ranging in the set $\{25,35,40\}$.

(iv) the maximum lag $m l$ for the matrix $\mathbf{A}^{k}(L)$ : ranging from 1 to 5 .

(v) the size $l$ of the rolling window: ranging from 5 to 12 years.

By selecting the values of the parameters which guarantee the lowest mean RMSFE, the chosen configuration for the IP is the following:

$$
(r, k, d, m l, l)=(4, \text { Parzen, } 25,4,12) .
$$

Instead, the chosen configuration for the CPI is the following:

$$
(r, k, d, m l, l)=(2, \text { Rectangular }, 25,1,7) .
$$

\section{Results on the proper sample}

\subsection{Prediction of the Industrial Production and the Inflation}

Now, the forecasting performances of the three dynamic factor models over the IP and CPI are compared on the proper sample, which starts on January 2001 and ends on November 2015. To forecast the IP, we changed the size of the rolling window employed in SW from 7 to 12 years. Instead, to forecast the CPI, we changed the size of the rolling window employed in SW from 12 to 7 years. Hence, to forecast the IP and the CPI, all dynamic factor models employ a rolling window of the same length. The common benchmark for the factor models is the autoregressive process (AR) of order four. In table 1, the average RMSFEs (relative to the AR) of the selected dynamic factor models are reported for the IP and CPI on the whole proper sample. However, as reported by CEPR, during the proper sample, the european economy faces two crisis periods: the first starts on May 2008 and ends on January 2009. The second starts on September 2011 and ends on March 2013. Hence, it is reasonable to assess whether the relative forecasting performances of the three dynamic factor models present a relevant 


\section{Fabio Della Marra}

change during the crisis periods. In table 2, the average RMSFEs (relative to the AR) of the three dynamic factor models are reported for the IP and the CPI from January 2001 to April 2008 (i.e. before the first crisis on the proper sample). In table 3 , the average RMSFEs (relative to the AR) of the three dynamic factor models are reported for the IP and the CPI from January 2001 to August 2011 (i.e. before the second crisis on the evaluation sample). One, two or three asterisks indicate that the null of equal performance of the three factor models relative to AR is rejected at, respectively, the 1\%,5\%,10\% significance by the Giacomini-White Test. One, two or three daggers indicate that the null of equal performance of FHLR, FHLZ relative to SW is rejected at, respectively, the $1 \%, 5 \%, 10 \%$ significance by the Giacomini-White Test. For further details about Giacomini-White Test, see [15].

Table 1. RMSFEs on the whole sample: IP on the left, CPI on the right.

\begin{tabular}{|c|c|c|c|c|c|c|}
\hline & \multicolumn{3}{|c|}{ IP } & \multicolumn{3}{c|}{ CPI } \\
\hline$h /$ model & FHLZ & FHLR & SW & FHLZ & FHLR & $S W$ \\
\hline 1 & 0.97 & 0.95 & 0.92 & 0.95 & 1.01 & 1.17 \\
3 & 0.84 & 0.82 & 0.82 & $0.88^{* * *}$ & 0.95 & 1.08 \\
6 & 0.66 & 0.66 & 0.68 & 0.85 & 0.94 & 1.04 \\
12 & 0.80 & 0.81 & 0.79 & 0.85 & 0.87 & 1.18 \\
24 & 0.94 & 0.94 & 0.92 & 1.02 & 1.02 & 1.47 \\
\hline
\end{tabular}

Table 2. RMSFEs from January 2001 to April 2008: IP on the left, CPI on the right.

\begin{tabular}{|c|c|c|c|c|c|c|}
\hline & \multicolumn{3}{|c|}{ IP } & \multicolumn{3}{c|}{ CPI } \\
\hline$h /$ model & FHLZ & FHLR & $S W$ & FHLZ & FHLR & $S W$ \\
\hline 1 & 1.15 & 1.20 & 1.24 & 0.96 & 0.98 & $0.99^{* * *}$ \\
3 & 0.80 & 0.82 & 0.79 & 1.05 & 1.07 & 1.14 \\
6 & 0.92 & 1.03 & 1.15 & $1.14^{* * \dagger}$ & $1.17^{* * \dagger \dagger}$ & $1.32^{*}$ \\
12 & 0.91 & 1.03 & 1.03 & $1.00^{* * * \dagger}$ & $1.04^{* * \dagger \dagger}$ & $1.32^{*}$ \\
24 & 0.85 & $0.84^{*}$ & $0.86^{*}$ & 0.79 & 0.82 & 1.09 \\
\hline
\end{tabular}

The relative performances of all methods tend to improve especially after the first crisis at horizons $h \in\{1,3,6,12\}$. This holds both for IP and CPI. This is also illustrated in figure 1 (on the left), in which the graphs of the cumulated sums of the square forecast error at $h=6$ for the IP are reported. The corresponding plots are similar for different values of $h \neq 24$ and are not reported here. The shaded areas correspond to the two crisis periods in the proper sample, according to CEPR. A relevant jump can be observed during the first crisis for all methods, including the benchmark, followed by a period of permanent flatness. However, after the first crisis, 


\section{Forecasting performance comparison of dynamic factor models}

Table 3. RMSFEs January 2001 to August 2011: IP on the left, CPI on the right.

\begin{tabular}{|c|c|c|c|c|c|c|}
\hline & \multicolumn{3}{|c|}{ IP } & \multicolumn{3}{c|}{ CPI } \\
\hline$h /$ model & FHLZ & FHLR & $S W$ & FHLZ & FHLR & $S W$ \\
\hline 1 & 0.95 & 0.87 & 0.82 & 0.97 & 1.06 & 1.24 \\
3 & 0.84 & 0.77 & 0.78 & 0.90 & 0.97 & 1.08 \\
6 & 0.65 & 0.65 & 0.66 & 0.85 & 0.92 & 0.97 \\
12 & 0.79 & 0.80 & 0.76 & 0.85 & 0.87 & 1.11 \\
24 & 0.98 & 0.98 & 0.93 & 1.24 & 1.23 & 1.82 \\
\hline
\end{tabular}

the sum of the cumulative forecast errors of AR increases substantially in comparison with those of the dynamic factor models (e.g., on October 2009, the sum of the cumulative forecast errors of AR increases, on average, more than $50 \%$ in comparison with those of the dynamic factor models). Similar results are obtained for the CPI and are not reported here.
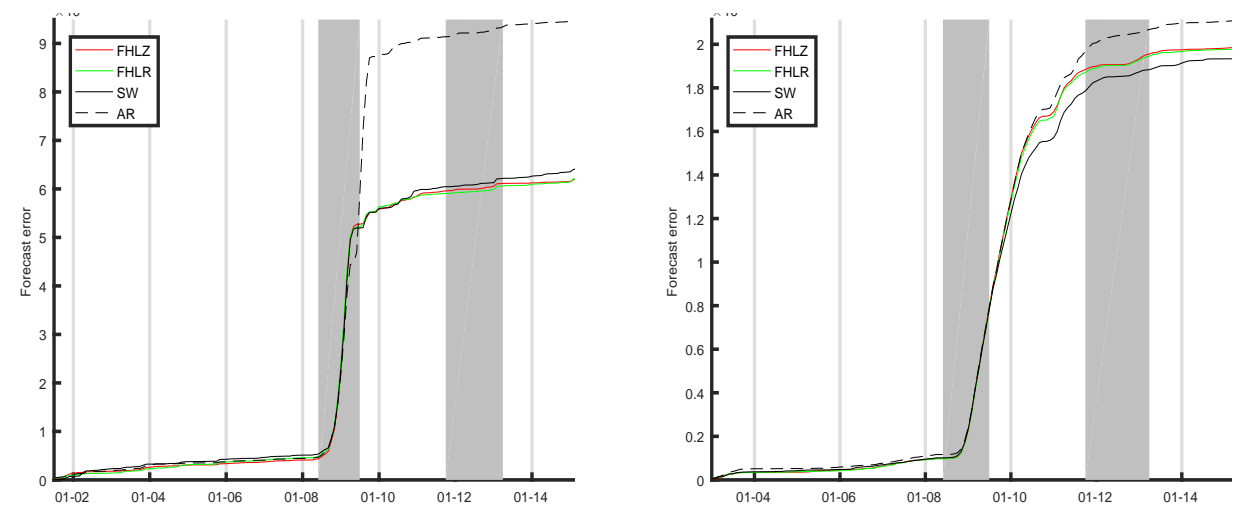

Figure 1. Cumulative sum of the square forecast error at $h=6$ for the IP (on the left). Cumulative sum of the square forecast error at $h=24$ for the IP (on the right).

Instead, at horizon $h=24$, the relative performances of all methods tend to worsen after the first crisis. This is also illustrated in figure 1 (on the right), in which the graph of the cumulated sums of the square forecast error for the IP are reported. In this plot, a jump during the first crisis can be obeserved whose slope is less remarked than in the left-side plot. This behaviour seems to persist until the beginning of the second crisis, after which a period of permanent flatness arises. However, no substantial increase in the sum of the cumulative forecast errors of AR appears in comparison with the dynamic factor models. Similar results are obtained for the CPI and are not reported here.

As in [11], to assess the forecasting performance of each couple of methods locally, each time series of the dataset is smoothed by a centered moving 


\section{Fabio Della Marra}

average of length $m=61$ (with coefficients equal to $1 / m$ ) and then the Fluctuation test ( [16]) is run, at $5 \%$ significance level. The results for the IP at horizons $h \in\{6,12,24\}$ are reported in Figure 2. All methods outperform AR significatively from the first crisis on. At horizon $h=6$, FHLR and FHLZ outperforms SW on average on the whole sample, except during the first crisis. Instead, at horizons $h \in\{12,24\}$, SW outperforms FHLR and FHLZ from the first crisis on. FHLR tends to outperform FHLZ in the two crisis periods. Outside the crisis periods, FHLZ tends, on average, to outperfom FHLR at horizons $h \in\{6,12\}$. Instead, at horizon $h=24$, FHLR outperforms FHLZ from the first crisis on.

The results for the CPI at horizons $h \in\{6,12,24\}$ are reported in Figure 3. FHLR and FHLZ tend to outperfom SW at all horizons, except FHLR at horizon $h=6$ during the former crisis.

FHLR and FHLZ tend to outperfom SW at all horizons, except FHLR at horizon $h=6$ during the first crisis. FHLR and FHLZ outperform AR at horizons $h \in\{6,12\}$. At horizon $h=24$, AR outperforms FHLR and FHLZ from the first crisis on. SW outperforms AR during the two crisis periods at horizons $h \in\{6,12\}$. At horizons $h \in\{12,24\}$, AR outperforms SW from the second crisis on. At all horizons, FHLZ outperforms FHLR during the first crisis. At horizons $h \in\{6,12\}$, this behaviour seems to be permanent. Instead, at horizon $h=24$, FHLR outperforms FHLZ from the second crisis on.

\subsection{Prediction of the dataset}

As in [11], this exercise has been extended to the other variables in the dataset. In table 4, we report the mean RMSFEs of each class of real (the first three) and nominal (the others) time series, taking AR as a benchmark. Similar results are obtained for the median and not reported here. The best performances are given in bold. We have excluded the categories "Demand", "Money" and "Exchange Rates', since AR outperformed all factor models at any horizon.

AR outperforms all methods at horizon $h=1$ for all cathegories, except for Interest Rates. Also, AR generally outperforms the dynamic factor models at shorter horizons (within 6 months) and it is the best methods also at $h=12$ for Unemployment and Wages. As to the factor models, FHLZ seem to be the most accurate methods for real variables. Instead, no method seems to be sistematically the most performing in forecasting the nominal variables. In any case, dynamic methods seem to produce more precise forecasts than SW both on nominal and real variables. In table 5, the distribution of the RMSE of the dynamic models for each cathegories 
Forecasting performance comparison of dynamic factor models
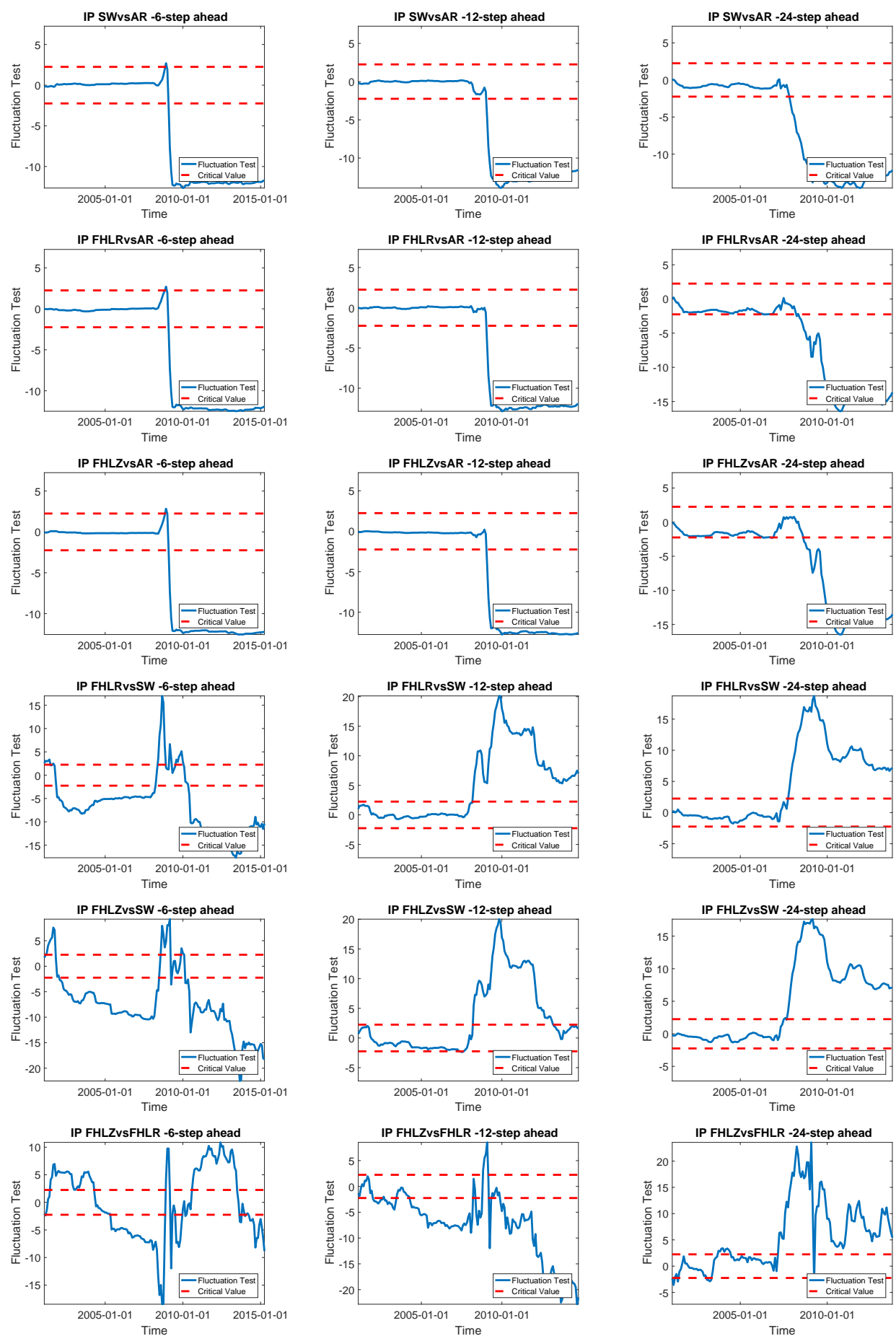

Figure 2. Fluctuation test for the IP. 
Fabio Della Marra
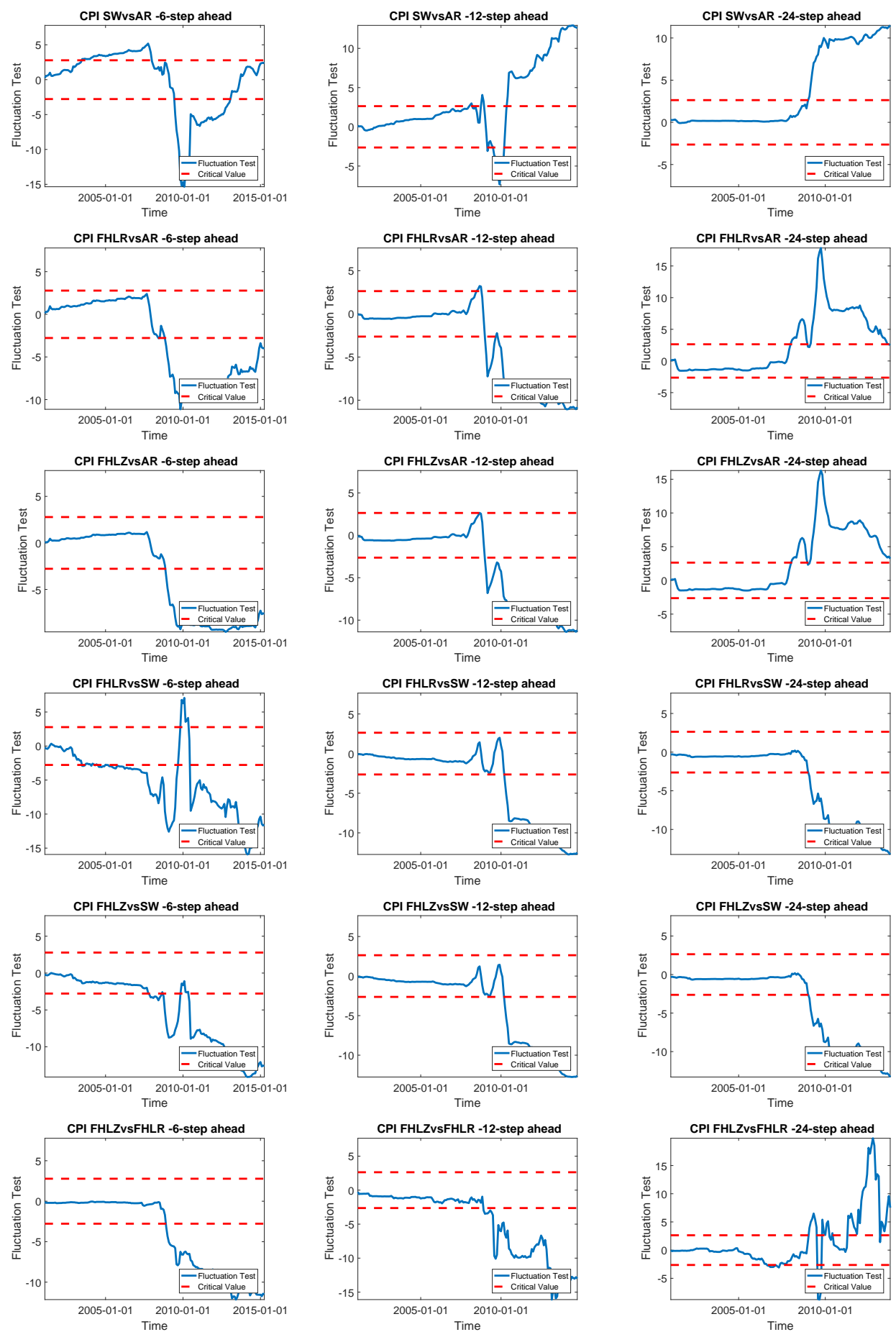

Figure 3. Fluctuation test for the CPI. 


\section{Forecasting performance comparison of dynamic factor models}

Table 4. Mean RMSFE for category.

\begin{tabular}{|c|c|c|c|c|c|}
\hline \multicolumn{7}{|c|}{ SW } \\
\hline \multicolumn{7}{|c|}{} \\
\hline Import-Export & 1.20 & 0.94 & 1.00 & 0.99 & 1.04 \\
Unemployment & 1.28 & 1.17 & 1.16 & 1.10 & 1.00 \\
Industrial Production & 1.12 & 1.01 & 0.93 & $\mathbf{0 . 9 2}$ & $\mathbf{0 . 9 6}$ \\
Prices & 1.01 & $\mathbf{0 . 9 7}$ & $\mathbf{0 . 9 6}$ & 1.01 & 1.16 \\
Wages & 1.19 & 1.28 & 1.39 & 1.33 & 1.31 \\
Surveys & 1.12 & 1.09 & 1.06 & $\mathbf{0 . 9 4}$ & $\mathbf{0 . 9 4}$ \\
Interest Rates & 1.06 & 1.11 & 1.08 & 1.06 & 1.00 \\
Stock Prices & 1.12 & 1.10 & 1.07 & 1.01 & 1.03 \\
\hline
\end{tabular}

\begin{tabular}{|c|c|c|c|c|c|}
\hline \multicolumn{7}{|c|}{ FHLR } \\
\hline & $h=1$ & $h=3$ & $h=6$ & $h=12$ & $h=24$ \\
\hline Import-Export & 1.18 & $\mathbf{0 . 9 3}$ & 0.97 & 0.99 & 1.00 \\
Unemployment & 1.29 & 1.17 & 1.15 & 1.06 & 0.99 \\
Industrial Production & 1.14 & 1.00 & 0.93 & 0.94 & 0.98 \\
Prices & 1.19 & 1.17 & 1.11 & 1.04 & 1.11 \\
Wages & 1.07 & 1.09 & 1.14 & 1.02 & $\mathbf{0 . 9 8}$ \\
Surveys & 1.14 & 1.12 & 1.04 & 1.01 & 1.09 \\
Interest Rates & 0.94 & 0.93 & 0.91 & $\mathbf{0 . 8 9}$ & $\mathbf{0 . 8 4}$ \\
Stock Prices & 1.01 & 1.01 & $\mathbf{0 . 9 9}$ & 1.01 & $\mathbf{0 . 9 9}$ \\
\hline
\end{tabular}

\begin{tabular}{|c|c|c|c|c|c|}
\hline \multicolumn{6}{|c|}{ FHLZ } \\
\hline & $h=1$ & $h=3$ & $h=6$ & $h=12$ & $h=24$ \\
\hline Import-Export & 1.16 & 0.97 & 0.96 & 0.97 & 1.00 \\
\hline Unemployment & 1.22 & 1.09 & 1.07 & 1.02 & 0.97 \\
\hline Industrial Production & 1.11 & 0.99 & 0.91 & 0.92 & 0.98 \\
\hline Prices & 1.11 & 1.11 & 1.10 & 1.10 & 0.92 \\
\hline Wages & 1.36 & 1.49 & 1.55 & 1.48 & 1.57 \\
\hline Surveys & 1.06 & 1.09 & 1.06 & 1.09 & 0.99 \\
\hline Interest Rates & 0.78 & 0.73 & 0.80 & 0.91 & 1.08 \\
\hline Stock Prices & 1.13 & 1.14 & 1.13 & 1.22 & 1.29 \\
\hline
\end{tabular}

are reported. The configuration of the parameters is the one chosen in the calibration process for the forecasting of the IP. Similar results are obtained for the configurations adopted for the CPI and are not reported here.

FHLZ is the only method which improves for at least half of the series More precisely, FHLZ is as accurate as AR till half of the series at horizon $h=1$. At the other horizons, it is as accurate as AR till the 75 -th percentile. $\mathrm{SW}$ is outperformed by frequency domain methods at all horizons and at 
Fabio Della Marra

Table 5. Distribution of the RMSFE.

\begin{tabular}{|c|c|c|c|c|c|}
\hline \multicolumn{7}{|c|}{ SW } \\
\hline Percentile & 0.05 & 0.25 & 0.50 & 0.75 & 0.95 \\
\hline$h=1$ & 0.89 & 1.02 & 1.08 & 1.17 & 1.53 \\
$h=3$ & 0.83 & 0.97 & 1.05 & 1.11 & 1.41 \\
$h=6$ & 0.81 & 0.96 & 1.03 & 1.13 & 1.39 \\
$h=12$ & 0.80 & 0.92 & 0.99 & 1.10 & 1.38 \\
$h=24$ & 0.82 & 0.94 & 1.03 & 1.14 & 1.39 \\
\hline
\end{tabular}

\begin{tabular}{|c|c|c|c|c|c|}
\hline \multicolumn{7}{|c|}{ FHLR } \\
\hline Percentile & 0.05 & 0.25 & 0.50 & 0.75 & 0.95 \\
\hline$h=1$ & 0.91 & 0.98 & 1.04 & 1.14 & 1.48 \\
$h=3$ & 0.83 & 0.94 & 1.02 & 1.07 & 1.30 \\
$h=6$ & 0.82 & 0.95 & 1.01 & 1.07 & 1.30 \\
$h=12$ & 0.85 & 0.96 & 1.01 & 1.05 & 1.25 \\
$h=24$ & 0.88 & 0.96 & 1.00 & 1.05 & 1.19 \\
\hline
\end{tabular}

\begin{tabular}{|c|c|c|c|c|c|}
\hline \multicolumn{7}{|c|}{ FHLZ } \\
\hline Percentile & 0.05 & 0.25 & 0.50 & 0.75 & 0.95 \\
\hline$h=1$ & 0.91 & 0.98 & 1.02 & 1.10 & 1.43 \\
$h=3$ & 0.85 & 0.95 & 0.99 & 1.07 & 1.28 \\
$h=6$ & 0.80 & 0.94 & 0.97 & 1.03 & 1.23 \\
$h=12$ & 0.83 & 0.93 & 0.97 & 1.01 & 1.17 \\
$h=24$ & 0.86 & 0.95 & 0.98 & 1.04 & 1.16 \\
\hline
\end{tabular}

almost all percentiles. Within frequency domain methods, FHLZ performs the best at 5-th and 95-th percentile.

\section{Conclusions}

In this paper, the forecasting performances of SW, FHLR and FHLZ are compared on a EU macroeconomic dataset which spans from February 1986 to November 2015. The EU dataset is split into two parts: the former is called calibration set and is used to tune the parameters of the dynamic factor models. The latter is called proper sample and is used to compare the forecasting performances of the three dynamic factor models. In the proper sample, all methods outperform AR after the first crisis at horizons $h \neq 24$. Instead, at horizon $h=24$, all methods tend to lose ground against AR after the first crisis. Moreover, FHLZ generally outperforms all methods on the prediction of the CPI. Instead, no method seems to outperform in 


\section{Forecasting performance comparison of dynamic factor models}

forecasting the IP, but all dynamic factor models outperform the benchmark AR. In the forecasting the whole dataset exercise, FHLZ is the most performing method in predicting real variables, whereas no significant evidencies appear on the prediction of nominal variables. However, apart from Interest Rates, all methods seem to perform poorly in comparison with AR.

\section{Acknowledgements}

We would like to thank Donatella Albano, Alessandro Giovannelli, Fabrizio Laurini, Marco Lippi, Simona Sanfelici, Stefano Soccorsi and an anonimous referee for their support and comments on an earlier version of the manuscript.

\section{Appendix: Dataset and transformation}

In table A.1, the series which compose the dataset are reported. T code identifies the tranformation (further details are given below) and Des identifies the deseasonalized flag ( 1 if the series is deseasonalized, 0 otherwise). The Transformation Codes, Tcode, in table A.1, are defined as reported below:

$$
\begin{aligned}
& z_{i t}=x_{i t} \delta\left(T_{\text {code }}-1\right)+\left[(1-L) x_{i t}\right] \delta\left(T_{\text {code }}-2\right) \\
& +\left[(1-L)^{2} x_{i t}\right] \delta\left(T_{\text {code }}-3\right)+\ln \left(x_{i t}\right)\left(T_{\text {code }}-4\right) \\
& +(1-L) \ln \left(x_{i t}\right) \delta\left(T_{\text {code }}-5\right)+(1-L)^{2} \ln \left(x_{i t}\right) \delta\left(T_{\text {code }}-6\right) \\
& +(1-L)\left(1-L^{12}\right) \ln \left(x_{i t}\right) \delta\left(T_{\text {code }}-7\right)
\end{aligned}
$$

where $x_{i t}$ represents the raw time series $x_{i}$ at time $t$ and $\delta($.$) the Dirac$ delta function. 
Table A1: List of the time series.

\begin{tabular}{|c|c|c|c|c|}
\hline & Name & Long Desc. & Tcode & Des \\
\hline 1 & BDM1....A & BD MONEY SUPPLY - M1 - CURA & 6 & 1 \\
\hline 2 & $\mathrm{BDM} 2 \mathrm{C} \ldots \mathrm{B}$ & BD MONEY SUPPLY - M2 CURA & 6 & 0 \\
\hline 3 & BDM3C...B & MONEY SUPPLY - M3 - CURA & 6 & 0 \\
\hline 4 & FRM1....A & FR MONEY SUPPLY - M1 - CURN & 6 & 1 \\
\hline 5 & FRM $2 \ldots . \mathrm{A}$ & FR MONEY SUPPLY - M2 - CURN & 6 & 1 \\
\hline 6 & FRM $3 \ldots \mathrm{A}$ & FR MONEY SUPPLY - M3 - CURN & 6 & 1 \\
\hline 7 & ITM1....A & IT MONEY SUPPLY: M1 - CURN & 6 & 1 \\
\hline 8 & ITM $2 \ldots . . A$ & IT MONEY SUPPLY: M2 - CURN & 6 & 1 \\
\hline 9 & ITM $3 \ldots . . A$ & MONEY SUPPLY: M3 - CURN & 6 & 1 \\
\hline 10 & NLM1....A & NL MONEY SUPPLY - M1 - CURN & 6 & 1 \\
\hline 11 & NLM $2 \ldots . . \mathrm{A}$ & NL MONEY SUPPLY - M2 - CURN & 6 & 1 \\
\hline 12 & NLM3....A & NL MONEY SUPPLY - M3 - CURN & 6 & 1 \\
\hline 13 & EMECBM1.B & EM MONEY SUPPLY: M1 - CURA & 6 & 0 \\
\hline 14 & EMM $2 \ldots . . B$ & EM MONEY SUPPLY: M2 - CURA & 6 & 0 \\
\hline 15 & EMECBM3.B & EM MONEY SUPPLY: M3 - CURA & 6 & 0 \\
\hline 16 & NLIMPGDSA & NL IMPORTS - CIF - CURN & 5 & 1 \\
\hline 17 & NLEXPGDSA & NL EXPORTS - FOB - CURN & 5 & 1 \\
\hline 18 & FRIMPGDSB & FR IMPORTS FOB - CURA & 5 & 1 \\
\hline 19 & FREXPGDSB & FR EXPORTS FOB - CURA & 5 & 1 \\
\hline 20 & ESOXT003b & ES ITS EXPORTS F.O.B. TOTAL - CURA & 5 & 1 \\
\hline 21 & ESOXT009b & ES ITS IMPORTS C.I.F. TOTAL - CURA & 5 & 1 \\
\hline 22 & ESEXPGDSD & ES EXPORTS - CONA & 5 & 1 \\
\hline 23 & ESIMPGDSD & ES IMPORTS - CONA & 5 & 1 \\
\hline 24 & ESEXPPRCF & ES EXPORT UNIT VALUE INDEX - NADJ & 5 & 1 \\
\hline 25 & ESIMPPRCF & ES IMPORT UNIT VALUE INDEX - NADJ & 5 & 1 \\
\hline 26 & BDEXPGDSB & BD EXPORTS OF GOODS (FOB) - CURA & 5 & 1 \\
\hline 27 & BDIMPGDSB & BD IMPORTS OF GOODS (CIF) - CURA & 5 & 1 \\
\hline 28 & BDEXPPRCF & BD EXPORT PRICE INDEX - NADJ & 7 & 1 \\
\hline 29 & BDIMPPRCF & BD IMPORT PRICE INDEX - NADJ & 7 & 1 \\
\hline 30 & ITEXPPRCF & IT EXPORT UNIT VALUE INDEX - NADJ & 7 & 1 \\
\hline 31 & BDOCC011 & BD REAL EFFECTIVE EXCHANGE RATES - CPI BASED - NADJ & 5 & 0 \\
\hline 32 & BGOCC011 & BG REAL EFFECTIVE EXCHANGE RATES - CPI BASED - NADJ & 5 & 0 \\
\hline 33 & ESOCC011 & ES REAL EFFECTIVE EXCHANGE RATES - CPI BASED - NADJ & 5 & 0 \\
\hline 34 & FNOCC011 & FN REAL EFFECTIVE EXCHANGE RATES - CPI BASED - NADJ & 5 & 0 \\
\hline
\end{tabular}




\begin{tabular}{|c|c|}
\hline & 3D \\
\hline NAGES.F & ES WAGES: INCOME INDICATOR - VOLN \\
\hline ESWAGES\%F & ES WAGES: INCOME INDICATOR (\%YOY) - VOLN \\
\hline FRI..RELF & FR REAL EFFECTIVE FX RATE (REER) BASED ON UNIT LABOUR COSTS - NADJ \\
\hline ITI..RELF & IT REAL EFFECTIVE FX RATE (REER) BASED ON UNIT LABOUR COSTS - NADJ \\
\hline ITWAGES.F & IT CONTRACTUAL HOURLY WAGE: ALL WORKERS - NADJ \\
\hline ITOLC007H & IT HOURLY WAGE RATE: INDUSTRY INCL. CONSTRUCTION - PROXY NADJ \\
\hline ITWAGES\%F & IT CONTRACTUAL HOURLY WAGE: ALL WORKERS (\%YOY) - NADJ \\
\hline NLI..RELF & NL REAL EFFECTIVE FX RATE (REER) BASED ON UNIT LABOUR COSTS - NADJ \\
\hline $\mathrm{NLOLC007H}$ & NL HOURLY WAGE RATE: MFG - PROXY NADJ \\
\hline BDIPTOT.G & BD INDUSTRIAL PRODUCTION INCLUDING CONSTRUCTION (CAL ADJ) - VOLA \\
\hline BDESPISDH & BD IPI: MIG - DURABLE CONSUMER GOODS, VOLUME IOP (WDA) - VOLN \\
\hline BDESPIESH & BD IPI: MIG-CAPITAL GOODS, VOLUME INDEX OF PRODUCTION (WDA) - VOLN \\
\hline BDESPISNH & BD IPI: MIG - NON-DURABLE CONSUMER GOODS, VOLUME IOP (WDA) - VOLN \\
\hline ESIPINTGH & ES INDUSTRIAL PRODUCTION - INTERMEDIATE GOODS - VOLN \\
\hline ESIPINVSH & ES INDUSTRIAL PRODUCTION - CAPITAL GOODS - VOLN \\
\hline ESESIBASG & ES IPI: MANUFACTURE OF BASIC METALS, VOLUME IOP (WDA) - VOLA \\
\hline ESIPOMNPH & $\begin{array}{l}\text { ES INDUSTRIAL PRODUCTION - OTHER NON-METAL MINERAL PRODUCTS - } \\
\text { VOLN }\end{array}$ \\
\hline ESIPTOT.G & ES INDUSTRIAL PRODUCTION (WDA) - VOLA \\
\hline ITIPTOT.G & IT INDUSTRIAL PRODUCTION - VOLA \\
\hline NLIPTOT.G & NL INDUSTRIAL PRODUCTION EXCLUDING CONSTRUCTION - VOLA \\
\hline FRIPTOT.G & FR INDUSTRIAL PRODUCTION - VOLA \\
\hline EU18 & EK PRODUCTION - TOTAL INDUSTRY EXCL. CONSTRUCTION - VOLA \\
\hline BDNEWORDE & BD MANUFACTURING ORDERS - SADJ \\
\hline BDRVNCARP & BD NEW PASSENGER CAR REGISTRATIONS - VOLN \\
\hline BGACECARP & BG NEW PASSENGER CAR REGISTRATIONS - VOLN \\
\hline ESCAR ...O & ES REGISTRATIONS: PASSENGER CAR - VOLA \\
\hline FRCARREGO & FR NEW CAR REGISTRATIONS (CAL ADJ) -VOLA \\
\hline FRHCONMFD & FR HOUSEHOLD CONSUMPTION - MANUFACTURED GOODS - CONA \\
\hline FRHCONDGD & FR HOUSEHOLD CONSUMPTION - DURABLE GOODS - CONA \\
\hline ITNEWORDF & IT NEW ORDERS - NADJ \\
\hline ITRETTOTF & IT RETAIL SALES - NADJ \\
\hline BDCNFCONQ & BD CONSUMER CONFIDENCE INDICATOR - GERMANY - SADJ \\
\hline BGCNFCONQ & BG BNB CONS. SVY.: CONSUMER CONFIDENCE INDICATOR (EP) - SADJ \\
\hline BGCNFBUSQ & BG BUSINESS INDICATOR SURVEY - ECONOMY - SADJ \\
\hline BGEUSIOBQ & IND.: OVERALL - ORD BOOKS - SADJ \\
\hline
\end{tabular}




\section{Forecasting performance comparison of dynamic factor models}

\section{REFERENCES}

1. J. H. Stock and M. W. Watson, Forecasting using principal components from a large number of predictors, Journal of the American Statistical Association, vol. 97, no. 460, pp. 1167-1179, 2002a.

2. J. H. Stock and M. W. Watson, Macroeconomic forecasting using diffusion indexes, Journal of Business 83 Economic Statistics, vol. 20, no. 2, pp. 147-162, 2002b.

3. M. Forni, M. Hallin, M. Lippi, and L. Reichlin, The generalized dynamic factor model: Identification and estimation, Review of Economics and Statistics, vol. 82, no. 4, pp. 540 - 554, 2000.

4. M. Forni, M. Hallin, M. Lippi, and L. Reichlin, The generalized dynamic factor model: One-sided estimation and forecasting, Journal of the American Statistical Association, vol. 100, pp. 830 - 840, 2005.

5. M. Forni, M. Hallin, M. Lippi, and P. Zaffaroni, Dynamic factor model with infinite dimensional factor space: representation, Journal of Econometrics, vol. 185, pp. 359-371, 2015.

6. M. Forni, M. Hallin, M. Lippi, and P. Zaffaroni, Dynamic factor model with infinite dimensional factor space: Asymptotic analysis, tech. rep., EIEF Working Paper 2016/07, Einaudi Institute for Economics and Finance, 2016.

7. J. Boivin and S. Ng, Understanding and comparing factor-based forecasts, International Journal of Central Banking, vol. 1, no. 3, pp. 117 151, 2005.

8. A. D' Agostino and D. Giannone, Comparing alternative predictors based on large-panel factor models, Oxford bulletin of economics and statistics, vol. 74, no. 2, pp. 306-326, 2012.

9. C. Schumacher, Forecasting german gdp using alternative factor models based on large datasets, Journal of Forecasting, vol. 26, no. 4, pp. 271302, 2007.

10. A. H. den Reijer et al., Forecasting dutch gdp using large scale factor models, tech. rep., Netherlands Central Bank, Research Department, 2005.

11. M. Forni, A. Giovannelli, M. Lippi, and S. Soccorsi, Dynamic factor model with infinite dimensional factor space: forecasting, tech. rep., 


\section{Fabio Della Marra}

CEPR Discussion Paper No. DP11161, 2016.

12. M. Forni and M. Lippi, The generalized dynamic factor model: representation theory, Econometric theory, vol. 17, no. 06, pp. 1113-1141, 2001.

13. M. Hallin and M. Lippi, Factor models in high-dimensional time series: A time-domain approach, Stochastic Processes and their Applications, vol. 123, no. 7, pp. 2678-2695, 2013.

14. M. Forni, D. Giannone, M. Lippi, and L. Reichlin, Opening the black box: Structural factor models with large cross sections, Econometric Theory, vol. 25, no. 05, pp. 1319-1347, 2009.

15. R. Giacomini and H. White, Tests of conditional predictive ability, Econometrica, vol. 74, no. 6, pp. 1545-1578, 2006.

16. R. Giacomini and B. Rossi, Forecast comparisons in unstable environments, Journal of Applied Econometrics, vol. 25, no. 4, pp. 595-620, 2010. 